\title{
Im Botaniker-Paradies: Der Emei Shan in Sichuan
}

\author{
Barbara KnickManN
}

\begin{abstract}
The Emei Shan is a massif in Sichuan/China. Many buddhist monasteries were built there and the view still existing are now a touristic attraction. The mountain can be explored by cable car or via stairs with tens of thousands of steps. This is where the sino-himalayan and the sino-japanese floras meet. Therefore and due to the geological diversity and the pronounced relief the biodiversity is extremely high. Some typical plants of the different forest communities on the Emei Shan are presented. The observations are from an excursion in May 2017.
\end{abstract}

\section{Zusammenfassung}

Der Emei-Shan ist ein Gebirgsstock in Sichuan/China. Am Emei-Shan entstanden über die Jahrhunderte viele buddhistische Klöster, die wenigen heute noch existierenden sind eine touristische Attraktion. Der Berg kann mit Hilfe einer Seilbahn oder über Treppen mit Zehntausenden von Stufen erkundet werden. Hier treffen die sino-japanischen und sino-himalayischen Floren aufeinander. Daher und aufgrund der geologischen Vielfalt und des ausgeprägten Reliefs ist die Artenvielfalt außerordentlich hoch. Einige typische Pflanzen der verschiedenen Waldgesellschaften am Emei Shan werden vorgestellt. Die Beobachtungen stammen von einer Exkursion im Mai 2017.

\section{Charakterisierung und Lage}

Der Emei Shan ist ein Gebirgsstock in der chinesischen Provinz Sichuan. Er liegt im Grenzgebiet zwischen dem tibetischen Hochplateau und dem östlich davon gelegenen so genannten Roten Be- cken, etwa auf dem 30. Breitengrad, was in etwa der geografischen Breite von Kairo entspricht. Der Gebirgsstock erstreckt sich über ca. $600 \mathrm{~km}^{2}$ und hat vier Hauptgipfel, wovon der höchste 3099 m hoch ist. Der Höhenunterschied vom umgeben-

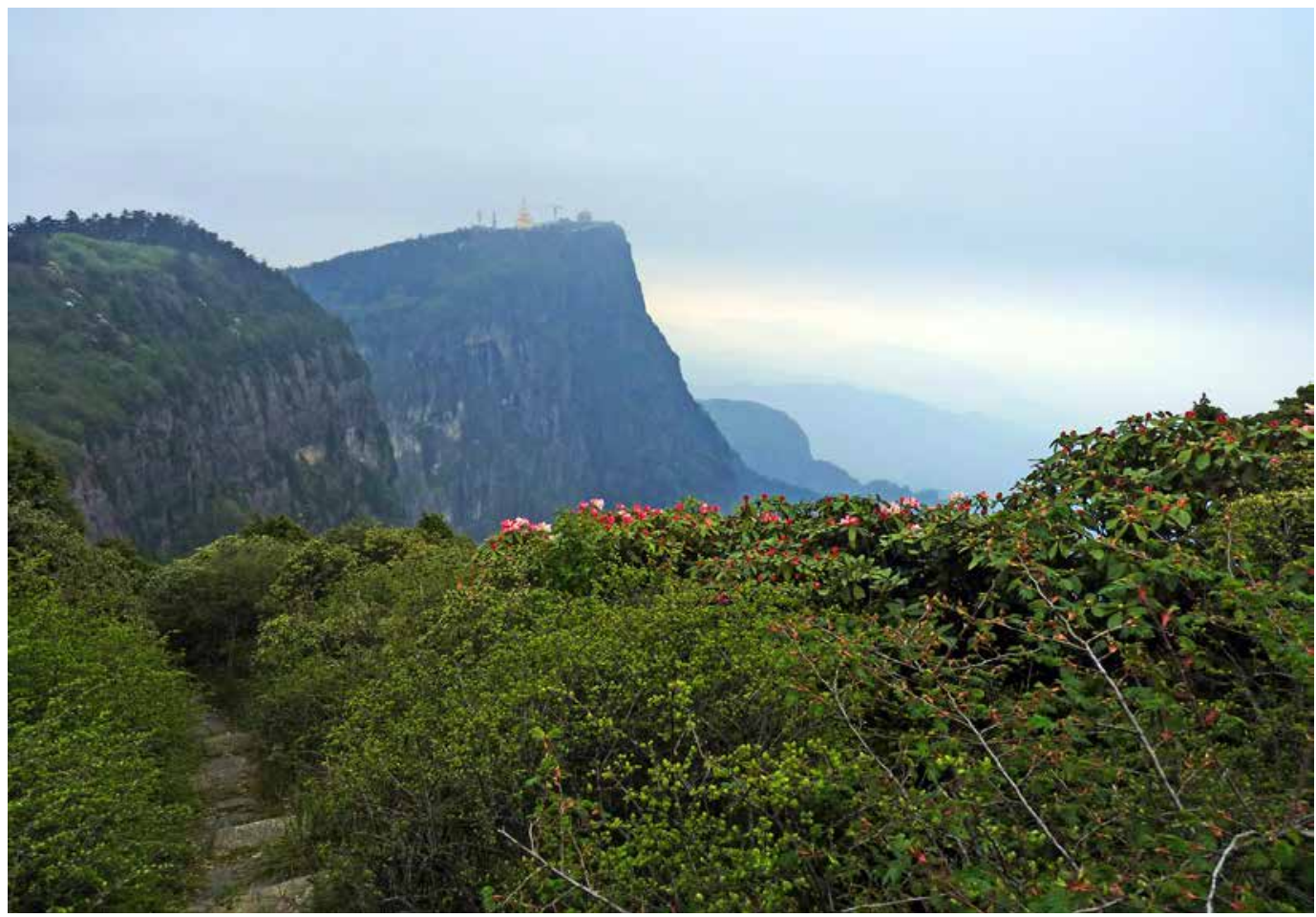

Abb. 1: Blick zum Golden Summit. (Foto: B. Knickmann) 
den Umland bis zum höchsten Gipfel beträgt rund $2600 \mathrm{~m}$.

\section{Klima}

Der Emei Shan liegt im humiden, subtropischen Monsunklima. In Höhenlagen unter $1500 \mathrm{~m}$ liegt die Jahresdurchschnittstemperatur bei $17,2{ }^{\circ} \mathrm{C}$. Es schließt sich zwischen $1500 \mathrm{~m}$ und $2100 \mathrm{~m}$ eine warm-temperierte Zone an, gefolgt von einer mittleren temperierten Zone $(2100 \mathrm{~m}-2500 \mathrm{~m})$. Oberhalb von $2500 \mathrm{~m}$ beträgt die Jahresdurchschnittstemperatur $3{ }^{\circ} \mathrm{C}$. Regen fällt ergiebig und durchschnittlich an 264 Tagen pro Jahr; am Bergfuß sind es durchschnittlich $1528 \mathrm{~mm}$, am Gipfel 1756 mm. Mit jährlich 323 Nebeltagen ist das Gebiet besonders nebelreich und damit die Luftfeuchtigkeit entsprechend hoch.

\section{Geologie und Relief}

Die Geologie des Emei Shan in einem kurzen Absatz zu beschreiben ist kaum möglich. Zum Verständnis des außergewöhnlichen floristischen Reichtums des Gebietes sei nur so viel gesagt:
Die geologischen Verhältnisse sind außerordentlich komplex. Sich kreuzende Verwerfungslinien verschiedenster Arten und Kategorien prägen die Geologie des Gebirgsstocks. Sediment- und Urgesteine wechseln sich ab, außerdem gibt es Gesteine vulkanischen Ursprungs (Basalt, v.a. am Gipfel). Der Emei Shan ist durchzogen von tiefen, u.a. durch Erosion bedingten Tälern, es gibt zahlreiche Höhlen. Die Reliefenergie ist gewaltig, immer wieder tun sich schwindelerregende Blicke über steile und hohe Klippen auf. Der Abfall vom zweithöchsten Gipfel nach Westen beträgt über $1600 \mathrm{~m}$.

\section{Artenreichtum am Emei Shan}

Der Emei Shan liegt an der Grenze zwischen der „sino-japanese“ und der „sino-himalayan“ Zone; Taxa dieser Regionen überlappen sich in ihren Vorkommensgebieten, u.a. deswegen ist die Artenzahl im Gebiet so enorm hoch. Die hohe Zahl an Taxa wird darüber hinaus durch die komplexen geologischen Verhältnisse, die Abfolge verschiedener Höhenstufen und damit Klimazonen sowie

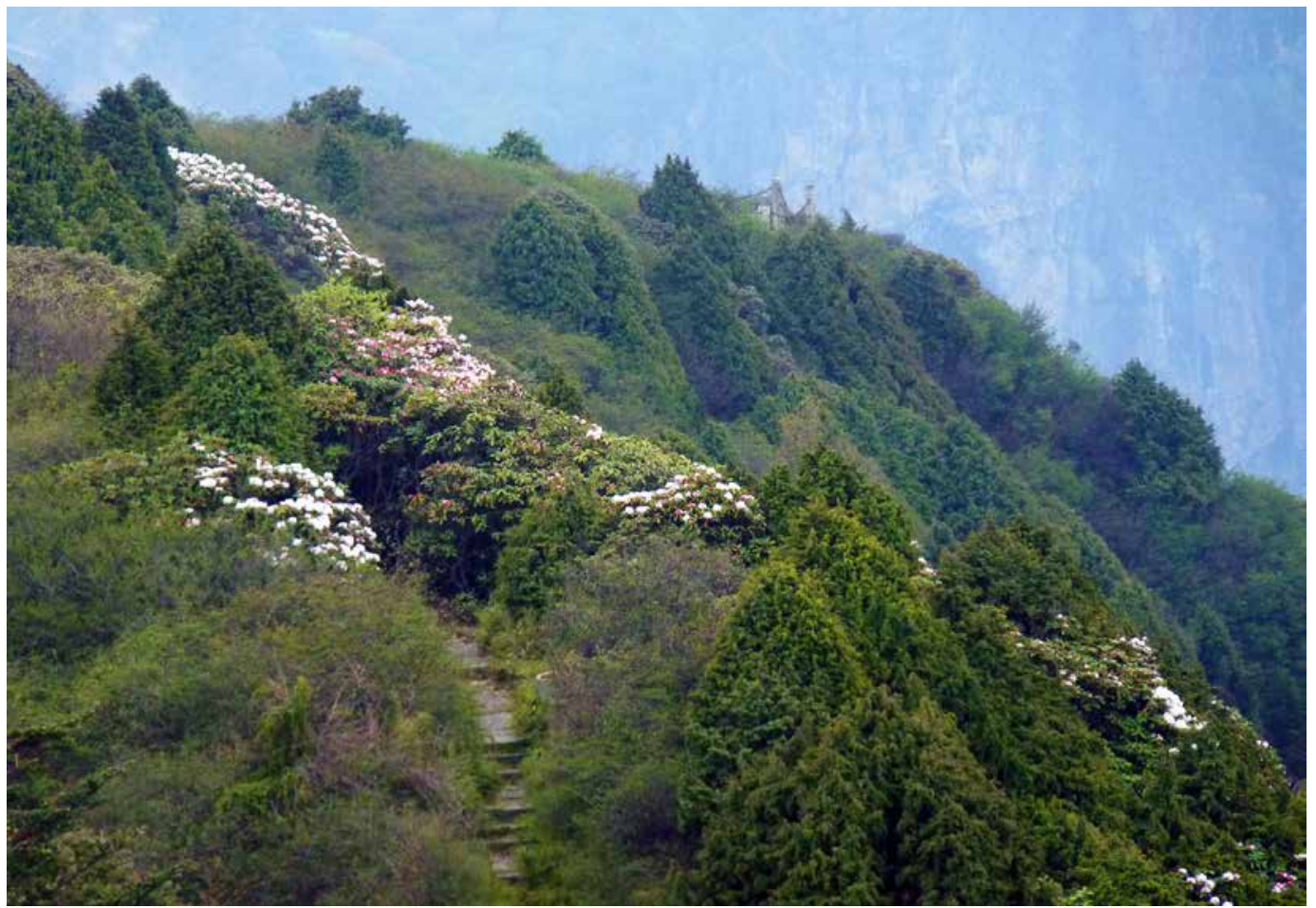

Abb. 2: Blick vom Huazang-Tempel. (Foto: B. Knickmann) 


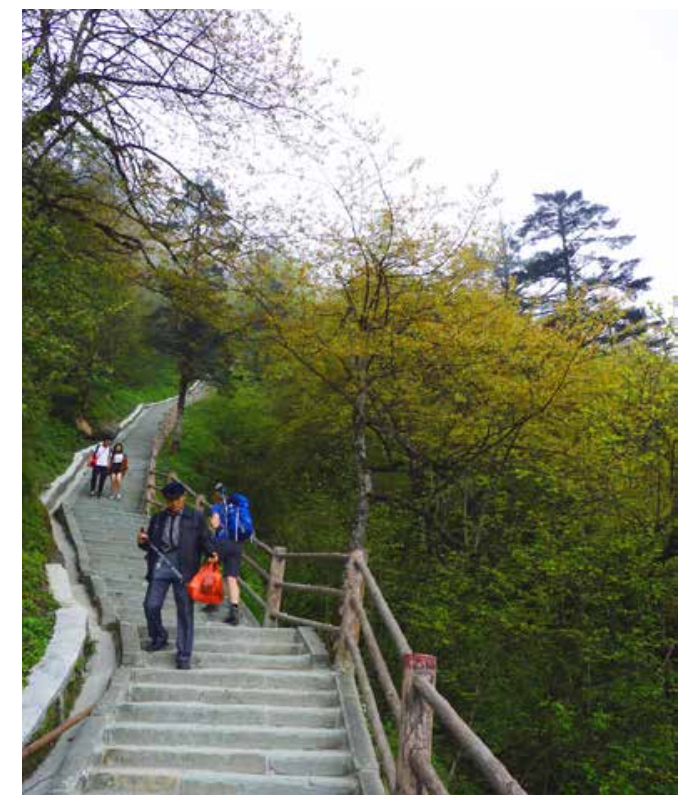

Abb. 3: Treppenweg oberhalb des Jieyin-Tempels.

(Foto: B. KNickmanN)

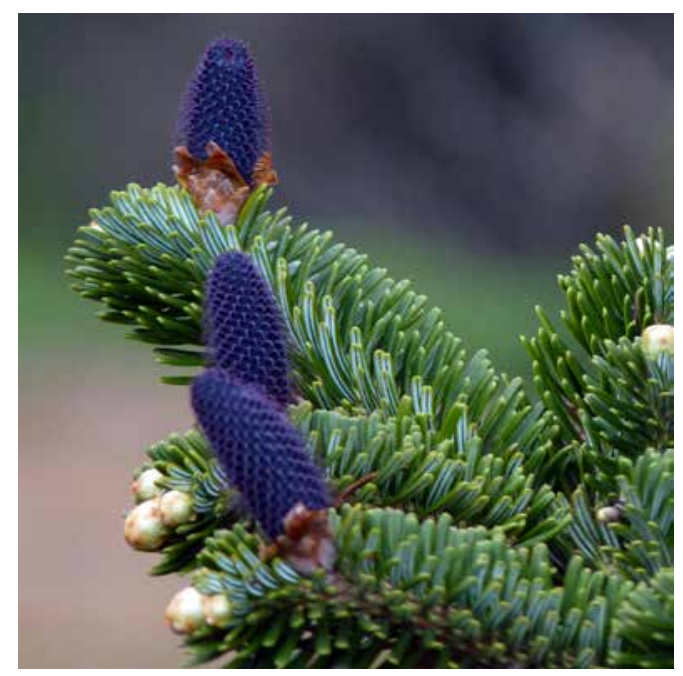

Abb. 4: Zweig von Abies fabri mit Zapfen.

(Foto: B. KNickMAnN)

die relativ ursprüngliche Vegetation bedingt. LI \& SHI (2007) geben in „Plants of Mount Emei“ die beeindruckende Zahl von 3703 Pflanzenarten (1271 Gattungen, 280 Familien) für den Emei Shan an. Davon sind allein 430 Farn-Arten in 105 Gattungen vertreten. Damit kommen am Emei Shan mehr als $10 \%$ der gesamten Flora Chinas

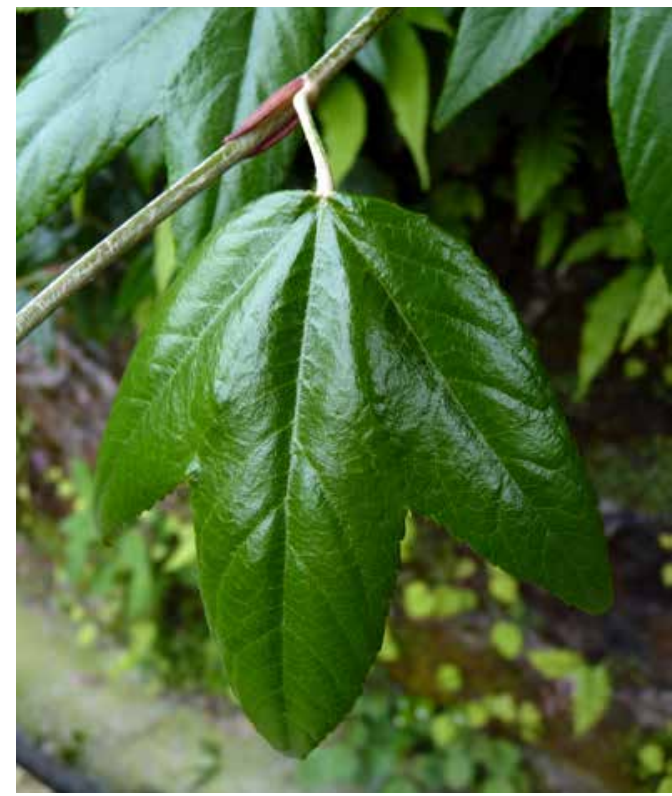

Abb. 5: Rubus henryi unterhalb Elephant Washing Temple. (Foto: B. Knickmann)

auf nur ca. 0,006 \% seiner Fläche vor! 106 der Taxa sind endemisch, viele von ihnen tragen den Art-Beinamen emeiensis bzw. omeiensis. Typisch für den Emei Shan sind ursprüngliche Vorkommen relativ vieler entwicklungsgeschichtlich sehr alter, primitiver Taxa, wie z.B. Liriodendron, Davidia, Tetracentron, Eucommia oder Euptelea. Außerdem ist die Flora des Emei Shan besonders reich an Medizinalpflanzen: 1655 Taxa sind medizinisch genutzt.

In diesem Artikel im Folgenden genannte Artenzahlen sind Li \& SHI (2007) entnommen.

\section{Religion, Kultur und Erschließung}

Um die kultur- wie naturhistorische Bedeutung des Emei Shan zu verstehen, ist ein Blick auf die geschichtliche Entwicklung hilfreich. Das Gebiet hat eine außerordentlich lange buddhistische bzw. daoistische Tradition. Seit dem 4. Jh. n. Chr. wurden dort zahlreiche Klöster gegründet, mit vielen Hundert Mönchen und Nonnen. Das erste buddhistische Kloster ganz Chinas stand am Emei Shan! Durch die politischen Entwicklungen in China im 20. Jahrhundert sind von diesen Klöstern nur noch knapp 30 vorhanden. Der Emei 


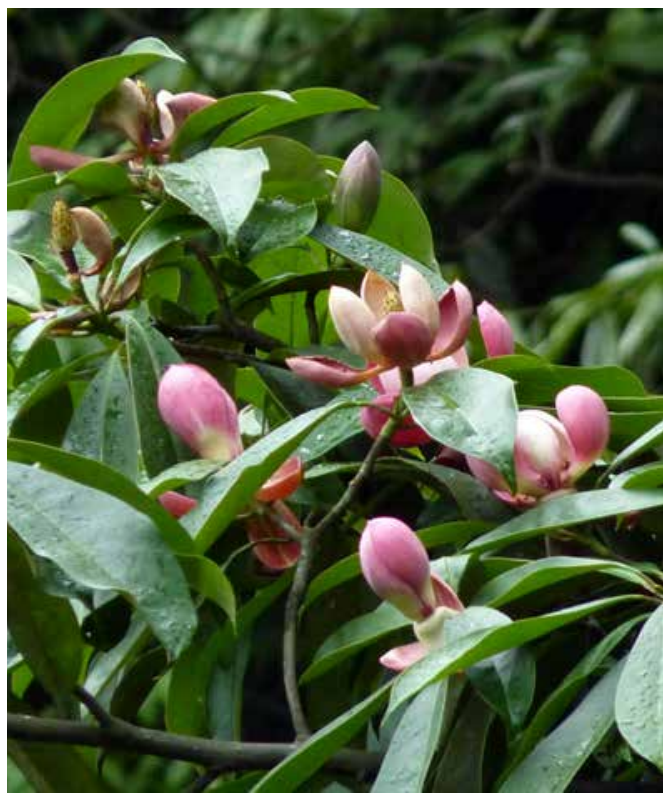

Abb. 6: Manglietia szechuanica. (Foto: B. Knickmann)

Shan zählt nach wie vor zu den vier heiligen chinesischen Bergen.

Reiseberichte aus vergangenen Jahrhunderten erzählen von den abenteuerlichen Pilgerwegen, die auf den Gipfel führten. Der früheste Bericht dieser Art stammt vom chinesischen Dichter FAN Chengda, der 1177 den Berg bestiegen hat und seine Erfahrungen und Eindrücke in Verse gegossen hat. Schon damals wurde der Berg von Pilgern besucht. Und schon damals war er zumindest zum Teil durch Treppenanlagen erschlossen. Auch heute noch ist das Gebiet durch einen befestigten Wanderweg erschlossen, der hauptsächlich aus Treppen besteht. Zwischen 18000 und 24000 Stufen sollen es sein, man läuft wie durch eine gigantische Parkanlage. Abseits dieser Treppenwege ist es praktisch unmöglich, sich im Gelände zu bewegen.

Aufgrund der kulturgeschichtlichen Bedeutung wurde das Gebiet 1996 zum UNESCO Weltkulturerbe erklärt. Während der Emei Shan zwischen 1966 und 1976 für Touristen gesperrt war, verlief die touristische Entwicklung seit den 1980er Jahren immer rasanter und wurde auch

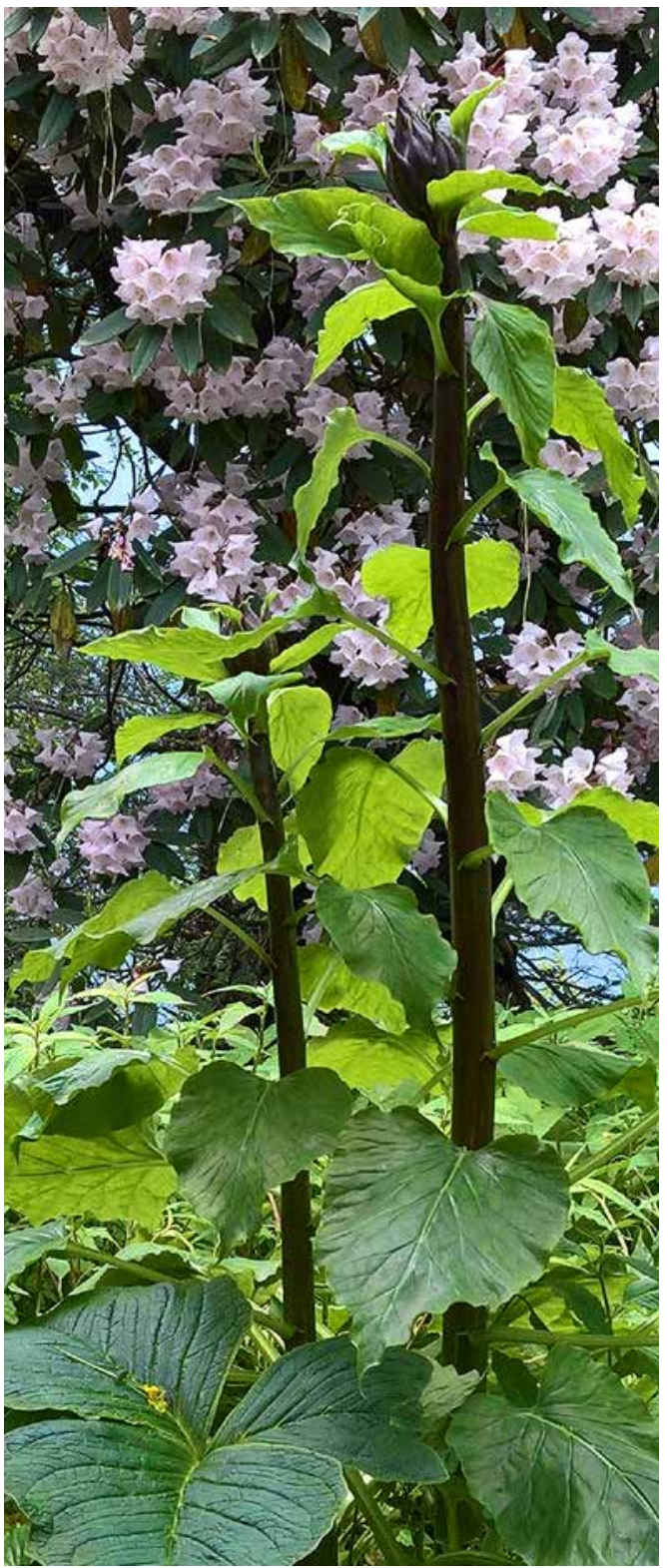

Abb. 7: Cardiocrinum giganteum unterhalb von Leidongping. (Foto: B. Knickmann)

zum erklärten (Staats-)Ziel. Ein Plan von 2005 erklärt den Emei Shan als Number One Mountain in China. Der Plan beinhaltet die Errichtung einer Beobachtungsterrasse mit riesiger, $48 \mathrm{~m}$ hoher, goldener, zehn-gesichtiger Samantabadhra-Statue am zweithöchsten Gipfel, dem so genannten Golden Summit des Emei Shan. Die Statue ist 


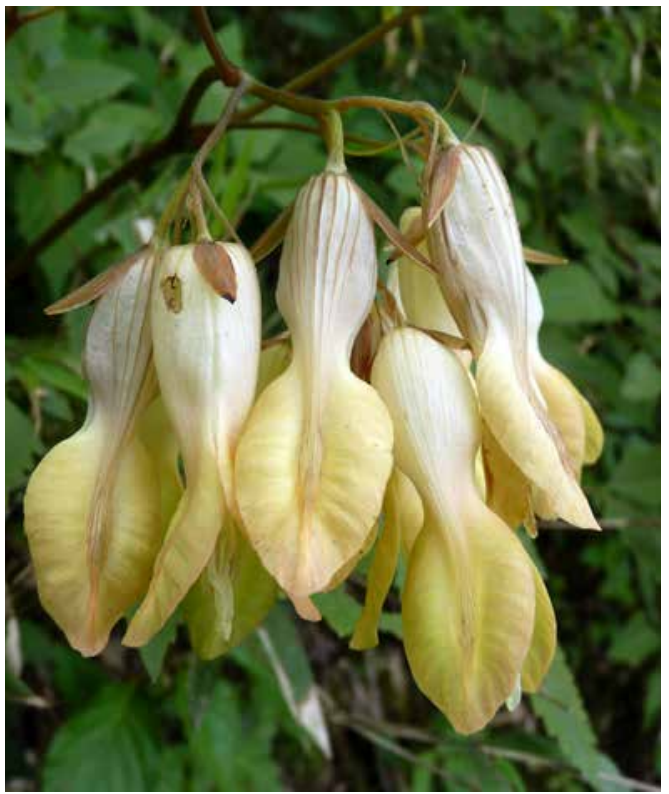

Abb. 8: Ichtyoselmis macrantha oberhalb von Leidongping. (Foto: B. Knickmann)

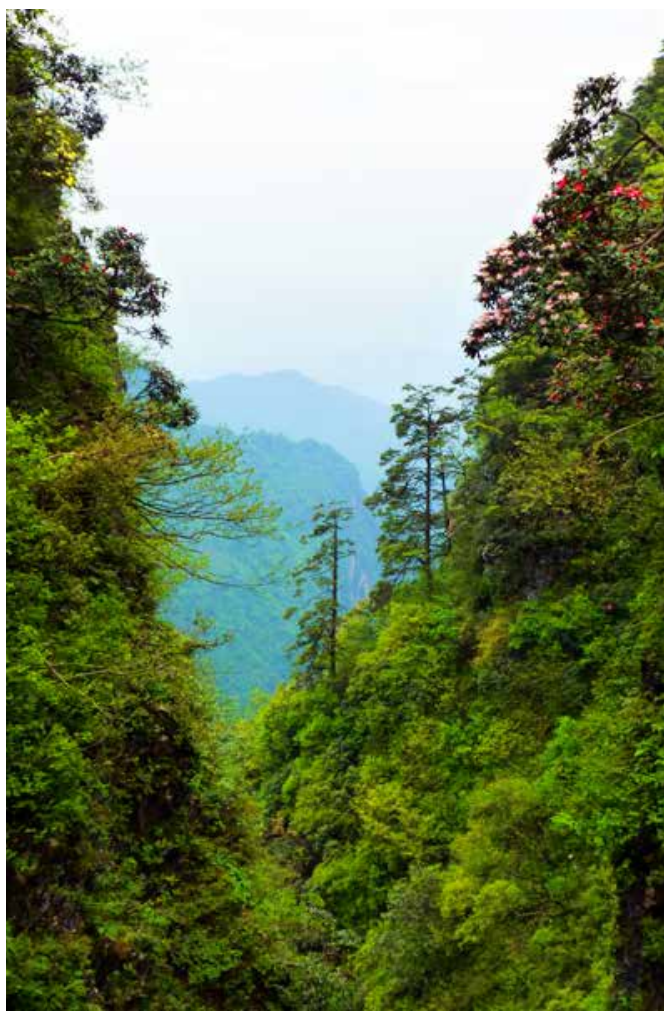

Abb. 9: Schlucht oberhalb Leidongping. (Foto: B. KNickManN) inzwischen gebaut und zeigt den größten Buddha aller chinesischen Buddhistischen Berge.

Nach der Wiederöffnung waren im Jahr 1977 etwa 100000 Besucher am Berg, 2015 waren es mehr als 3 Millionen Touristen. Der Ballungsraum rund um die Metropole Chengdu, einem der boomenden Wirtschaftszentren Westchinas mit mehr als 14 Millionen Einwohnern, ist nur eineinhalb Stunden Fahrtzeit vom Emei Shan entfernt. Es gibt eine Skipiste und eine Gondel, die die Gipfelregion erschließt. Bis zur Gondelstation gelangt man mit Shuttle-Bussen; so ist das Erreichen des Gipfels keineswegs mehr die alpinistische Herausforderung, wie sie es über Jahrhunderte war. Der Buddhismus am Berg hat überlebt, hat sich aber neu definiert. Die Klöster gehören zum touristischen Konzept und dienen u.a. der Unterkunft von Touristen.

\section{Erforschung der Flora am Emei Shan durch westliche Wissenschaftler}

Die erste Besteigung durch einen westlichen Wissenschaftler, von der eine Beschreibung vorliegt, erfolgte durch den englischen Orientalisten Edward Colborne Baber (1877), gefolgt von Alexander Hosie (1884). Weder Baber noch Hosie gehen in ihren Beschreibungen auf die Pflanzenwelt ein. Hosie zu Ehren wurde eine Gehölzart aus der Familie der Schmetterlingsblütler Ormosia hosiei genannt; die Art kommt in den Wäldern am Fuße des Emei Shan vor.

Eine zentrale Rolle bei der Erforschung des Berges kommt Augustine Henry zu. Henry war als britischer Zollbeamter in Sichuan stationiert, hatte v.a. mit chinesischen Medizinalpflanzen zu tun und war botanisch ein Autodidakt. Er war zwar nie selbst am Emei Shan, hat jedoch ERnst FABER (Besteigung 1887), Antwerp Edward Pratt (1890) und vor allem Ernest H. Wilson (1903) in entscheidendem Maße logistisch bei der floristischen Erforschung des Berges unterstützt. Auch der russische Botaniker Potanin erkundete das Gebiet (1893). Nach diesen Forschern benannte Taxa, die z.T. sehr prominent am Emei Shan vorkommen, sind Abies fabri, Sorbus prattii, Begonia wilsonii, Rubus henryi und Betula potaninii. 
Die Folge der Forschungs- und Sammelreisen zu Ende des 19. bzw. Beginn des 20. Jahrhunderts durch westliche Wissenschaftler ging mit LimPRICHT zu Ende, der 1914 am Emei Shan war. Aus den folgenden Jahrzehnten gibt es aufgrund politischer Wirren - zunächst in Europa, dann in China selbst - keine Berichte westlicher Forscher über das Gebiet. Im 20. Jahrhundert waren u.a. Roy Lancaster (1980) und Seamus O'Brien (2002) am Emei Shan unterwegs; von beiden Reisen gibt es beeindruckende Beschreibungen.

\section{Zonierung der Vegetation entlang des Höhengradienten}

Durch den großen Höhengradienten ergibt sich eine Abfolge von immergrünem Laubwald (evergreen broad-leaved forest zone) in der Höhenstufe zwischen $600 \mathrm{~m}$ und $1500 \mathrm{~m}$, gefolgt von einem Laub-Nadel-Mischwald (mixed forest zone) zwischen $1500 \mathrm{~m}$ und $2500 \mathrm{~m}$; ab $2500 \mathrm{~m}$ schließt sich bis zum Gipfel (3099 m) subalpiner Nadelwald (coniferous forest zone) an. Die für China außergewöhnlich gut erhaltene natürliche Vegetation mit verschiedenen Waldgesellschaften lässt sich v.a. durch die lange buddhistische Tradition erklären. Bäume wurden nur dann gefällt, wenn die aus Holz gebauten Klöster renovierungsbedürftig waren. Außerdem spielt sicher auch die schwierige Topografie am Berg eine Rolle, die eine Inkulturnahme für landwirtschaftliche Zwecke, aber auch eine forstliche Nutzung auf der weit überwiegenden Fläche ausschließt.

\section{Pflanzenarten der verschiedenen \\ Höhenstufen bzw. Waldgesellschaften}

Beginnend am Bergfuß folgt hier nun eine Beschreibung der Vegetationsstufen mit einer Auswahl typischer bzw. besonders auffälliger Arten. Die im Folgenden beschriebenen bzw. genannten Pflanzenarten wurden Ende Mai 2017 auf einer botanischen Exkursion am Emei Shan angetroffen.

In der Zone des immergrünen Laubwaldes wachsen besonders viele Vertreter der Familie der Lorbeergewächse, die in unterschiedlicher Artenzusammensetzung verschiedene Waldgesellschaften charakterisieren. Eine typische Baumart, die mit riesigen, angeblich 1000-jährigen Exemp-

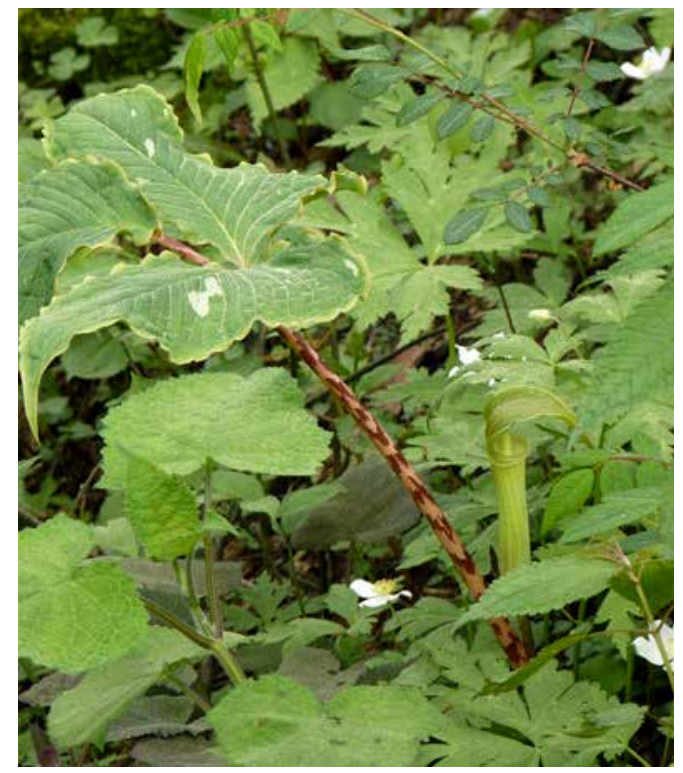

Abb. 10: Arisaema cf. lobatum. (Foto: B. Knickmann)

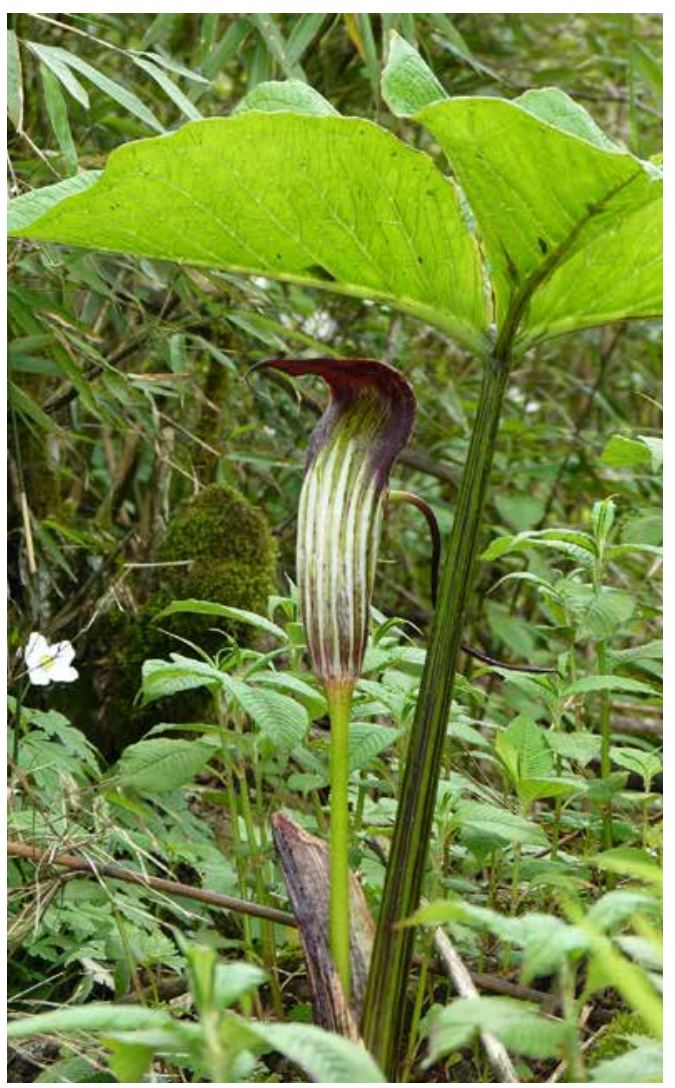

Abb. 11: Arisaema wilsonii bei Leidongpip.

(Foto: B. Knickmann) 


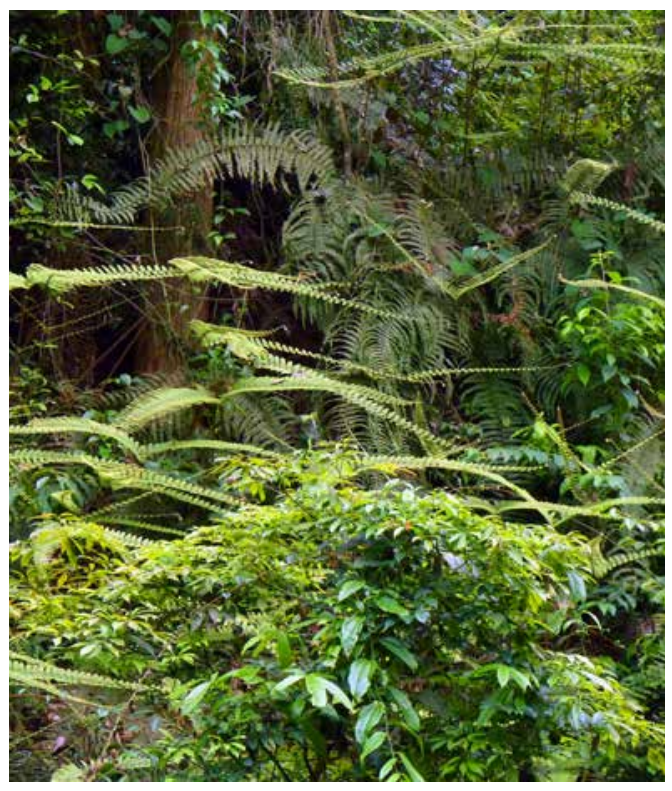

Abb. 12: Hicriopteris omeiensis. (Foto: B. KnICKManN) laren vorkommt, ist Phoebe zhenan. Die Gattung Lindera ist mit mehreren Arten vertreten, ebenso die Gattung Cinnamomum. Farne kommen hier besonders reichhaltig vor. Sie wachsen am Waldboden, aber auch epiphytisch (auf Bäumen) und epilithisch (auf/an Felsen) wie z.B. der ausschließlich auf Kalkgestein wachsende Pteris actiniopteroides mit seinen zweigestaltigen Wedeln. Auch die Spießtanne (Cunninghamia lanceolata) wächst in diesen Wäldern. Unter den krautigen Arten sind insbesondere verschiedene Brennnesselund Ingwergewächse häufig. In dieser Höhenstufe kommt auch die oben bereits erwähnte Begonia wilsonii vor. Die Unterseite ihrer Blätter ist leuchtend rot. Die Art braucht hohe Luftfeuchtigkeit. Der Emei Shan wird in der Flora of China als Verbreitungsgebiet ausdrücklich genannt. $B$. wilsonii ist eine von vier am Emei Shan vorkommenden Begonienarten. Die Kultur von Arten dieser Höhenstufe im Freiland ist in unseren Breiten-

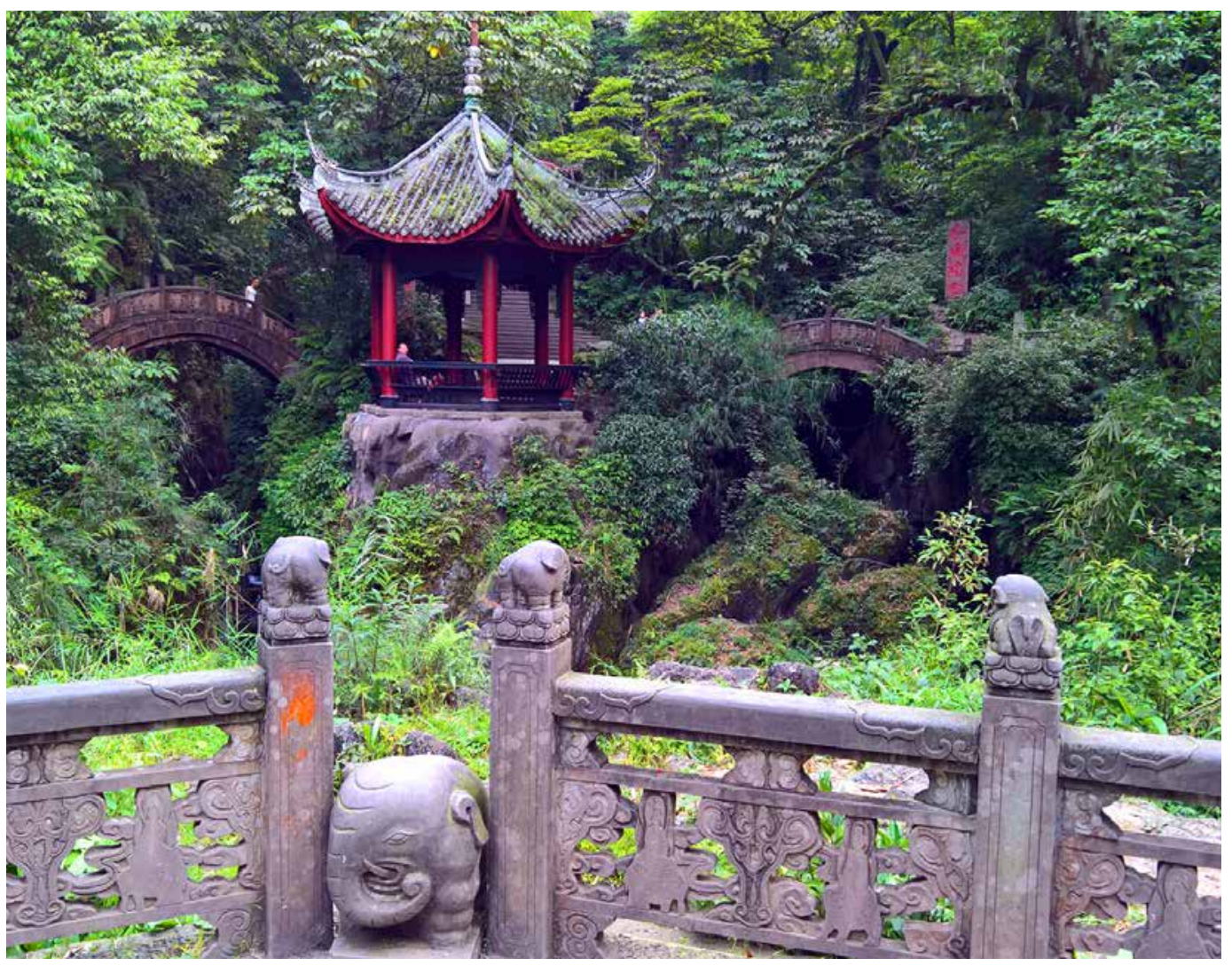

Abb. 13: Pavillon unterhalb Qinyin. (Foto: B. Knickmann) 
graden nur in besonders milden Lagen, nur mit Winterschutz oder nicht dauerhaft möglich.

In der Mischwaldzone in einer Höhenstufe von ca. $1500 \mathrm{~m}$ bis $2500 \mathrm{~m}$ dominieren u.a. verschiedene Acer-, Lithocarpus- und Castanopsis-Arten (vor allem Castanopsis platyacantha), jeweils aus der Familie der Buchengewächse, und der berühmte Taschentuchbaum (Davidia involucrata). Hier kommen auch einige seltene Magnoliengewächse vor, u.a. Manglietia szechuanica (= Magnolia figlarii). Diese Art wurde mit einem einzigen Individuum angetroffen, das sogar etikettiert war! $\mathrm{Zu}$ den Laubbäumen gesellt sich, besonders in höheren Lagen, schon Abies fabri, die dominante Nadelbaum-Art des Emei Shan. Eine weitere Nadelbaumart ist Tsuga chinensis.

Die Strauchschicht bilden eine Vielzahl unterschiedlichster Gattungen, unter den geläufigeren sind verschiedene Deutzien, es kommen sechs verschiedene Stachyurus-Arten vor, verschiedenste baum- oder strauchförmige Sorbus-Arten, die Gattung Cornus ist mit 13, Lonicera mit 12 Arten vertreten, Viburnum mit 25 Arten. Die Krautschicht ist ungeheuer artenreich. Einige bekannte Gartenpflanzen finden sich darunter, wie z.B. Iris japonica, Epimedium-Arten, Cardiocrinum giganteum oder Saxifraga stolonifera. Die seltene Bergenia emeiensis ist ein Beispiel für eine Art, deren ArtBeiname Bezug auf den Emei Shan nimmt. Diese Art war im Innenhof des Klosters Hongchungping als Zierpflanze verwendet. In Kultur kaum bekannt ist Ichtyoselmis macrantha (= Dicentra macrantha) aus der Familie der Mohngewächse (Papaveraceae). Sie wurde in verschiedenen Höhenlagen in stets sehr feuchten Waldpartien angetroffen. Ichtyoselmis ist eine monotypische Gattung, die von der Gattung Dicentra abgespalten wurde. Auch Meehania fargesii (Lamiaceae) wäre gartenwürdig, ist aber

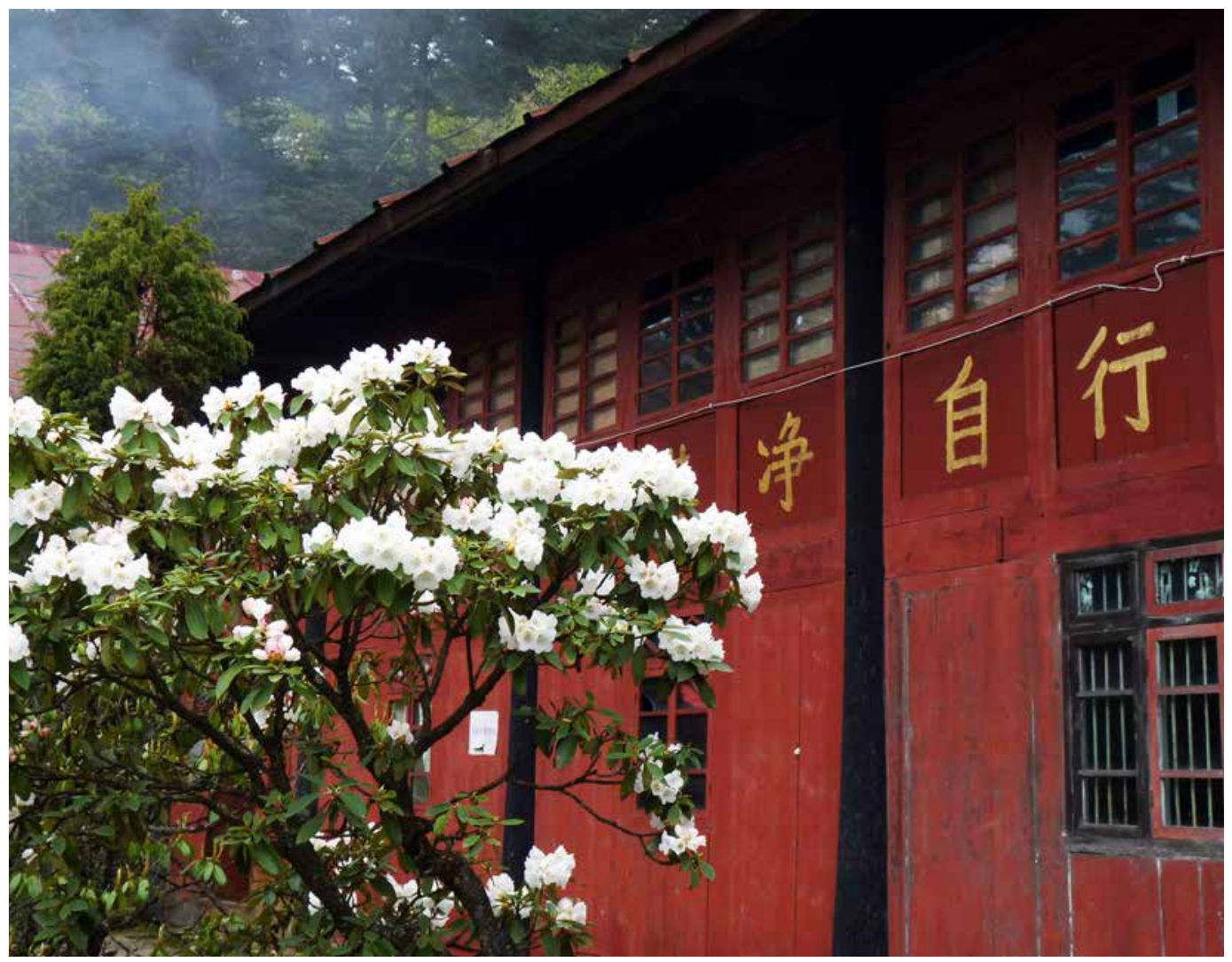

Abb. 14: Taiziping Monastery mit Rhododendron faberi. (Foto: B. KNICKManN) 


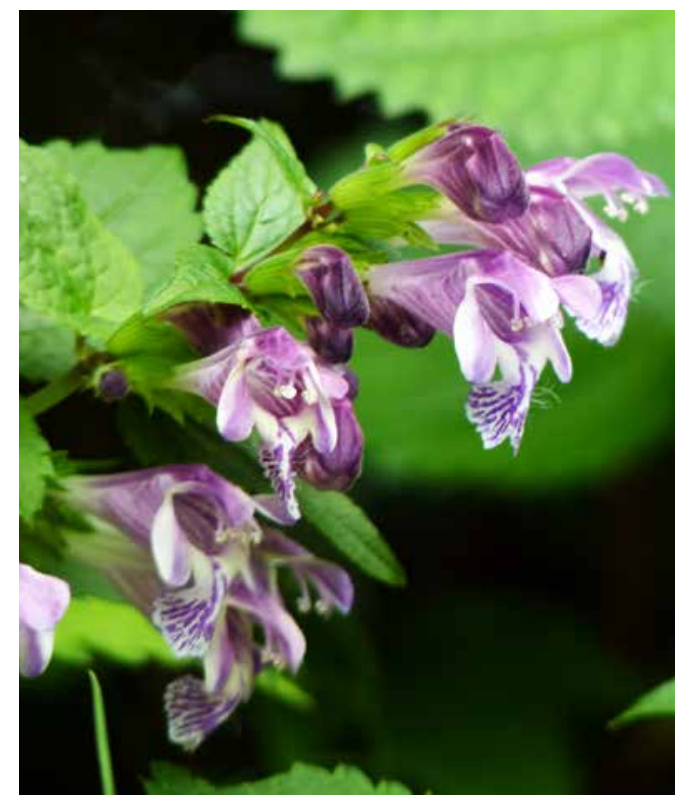

Abb. 15: Meehania fargesii oberhalb Elephant Washing Temple. (Foto: B. Knickmann)

kaum bekannt. Sie ist eine der vielen medizinisch (in diesem Fall gegen Durchfallerkrankungen) genutzten Arten, die am Emei Shan vorkommen. Liebhaber von Aronstabgewächsen kommen am Emei Shan voll auf ihre Kosten (es gibt allein 11 Arisaema-Arten, darunter die endemische Arisaema omeiense), genauso wie Rubus-Spezialisten (44 Arten).

In der Nadelwaldzone dominiert die bereits erwähnte Abies fabri, benannt nach Pastor ERnst FABER. Sie ist mir ihren blauen Zapfen eine ausgesprochen attraktive Tannen-Art, die in Kultur anspruchsvoll ist, muss man ihr doch erhebliche (Luft-)Feuchtigkeit bieten. Sie ist in den hohen Lagen des Emei Shan vergesellschaftet mit verschiedenen Rhododendren (u.a. Rhododendron faberi), Salix-, Spiraea- und Ribes-Arten. Abies fabri ist auf die Gebirge Sichuans beschränkt, wo sie prinzipiell bis in Höhen um 4000 m gedeiht. Der nach Osten vergleichsweise flache Gipfelbereich ist zudem dicht bewachsen von einer Bambusart, die in der Literatur als Arundinaria fangiana beschrieben wird. Krautige Pflanzen waren bei der Exkursion im Mai 2017 noch kaum in Blüte. Eine Ausnahme bildete Potentilla leuconota. Zwischen bunten Gebetszetteln leuchteten ihre gelben Blüten am Golden Summit des Emei Shan auf über 3000 m Höhe.

\section{Ausblick}

Es bleibt zu hoffen, dass eine weitere touristische Erschließung die naturräumlichen Besonderheiten des Emei Shan ausreichend berücksichtigt. Außerdem sollten Forderungen Gehör und Umsetzung finden, die einerseits besondere, nicht zugängliche Schutzgebiete am Berg umfassen, andererseits Gärtnereien propagieren, in denen volksmedizinisch nach wie vor genutzte und daher besonders gesuchte Arten kultiviert werden sollen, um die natürlichen Bestände der entsprechenden Arten am Berg zu schonen.

\section{Verwendete und weiterführende Literatur}

Bretschneider, E. 1898: History of european botanical discoveries in China. (2 Bände). - Reprint, Leipzig 1981

Grey-Wilson, C. 2011: Guide to the flowers of Western China. - Kew.

Hargett, J. M. 2007: Stairway to heaven: A journey to the summit of Mount Emei. - New York.

Lancaster, R. 2008: Plantsman's paradise. Travels in China. - Woodbridge.

Li, Z.-Y. \& SHI, L. (Hrsg.) 2007: Plants of Mount Emei. Peking.

O'Brien, S. 2011: In the footsteps of Augustine Henry. Garden Art Press. - Woodbridge.

Pratt, A. E. 1892: To the snows of Tibet through China. London.

Steiger, P. 2017: Eine botanische Pilgerreise. - Gartenpraxis 12/2017: 56-61

Zhu Zhengyin \& Luo Minghua 1997: Sustainable use and preservation of plant resources on Mt. Emei, Sichuan, China (https://www.nacsj.or.jp/pn/houkoku/h01-08/h08-no13.html)

\section{Anschrift der Autorin}

Barbara Knickmann, Sammlungsmanagement, Core Facility Botanischer Garten, Universität Wien, Rennweg 14, 1030 Wien, Österreich 